\title{
The Resistive-Plate WELL with Argon mixtures - A robust gaseous radiation detector
}

\author{
Luca Moleri ${ }^{\mathrm{a}, *}$, Fernando Domingues Amaro ${ }^{\mathrm{b}}$, Lior Arazi ${ }^{\mathrm{a}}$, Carlos Davide Rocha Azevedo ${ }^{\mathrm{c}}$, \\ Eraldo Oliveri ${ }^{\mathrm{d}}$, Michael Pitt ${ }^{\mathrm{a}}$, Jana Schaarschmidt ${ }^{\mathrm{a}}$, Dan Shaked-Renous ${ }^{\mathrm{a}}$, \\ Joaquim Marques Ferreira dos Santos ${ }^{b}$, João Filipe Calapez de Albuquerque Veloso ${ }^{c}$, \\ Amos Breskin ${ }^{a}$, Shikma Bressler ${ }^{a}$ \\ a Department of Particle Physics and Astrophysics, Weizmann Institute of Science, 76100 Rehovot, Israel \\ ${ }^{\mathrm{b}}$ LIBPhys, Department of Physics, University of Coimbra, RuaLarga, PT3004-516 Coimbra, Portugal \\ ${ }^{\mathrm{c}}$ I3N, Physics Department, University of Aveiro, 3810-193 Aveiro, Portugal \\ d CERN, Meyrin, Switzerland
}

\section{A R T I C L E I N F O}

\section{Article history:}

Received 15 March 2016

Accepted 1 June 2016

Available online 8 June 2016

Keywords:

Micropattern Gaseous detectors

THGEM

Calorimetery

\begin{abstract}
A B S T R A C T
A thin single-element THGEM-based, Resistive-Plate WELL (RPWELL) detector was operated with $150 \mathrm{GeV} / \mathrm{c}$ muon and pion beams in $\mathrm{Ne} /\left(5 \% \mathrm{CH}_{4}\right), \mathrm{Ar} /\left(5 \% \mathrm{CH}_{4}\right)$ and $\mathrm{Ar} /\left(7 \% \mathrm{CO}_{2}\right)$; signals were recorded with $1 \mathrm{~cm}^{2}$ square pads and SRS/APV25 electronics. Detection efficiency values greater than $98 \%$ were reached in all the gas mixtures, at average pad multiplicity of 1.2 . The use of the $10^{9} \Omega \mathrm{cm}$ resistive plate resulted in a completely discharge-free operation also in intense pion beams. The efficiency remained essentially constant at $98-99 \%$ up to fluxes of $\sim 10^{4} \mathrm{~Hz} / \mathrm{cm}^{2}$, dropping by a few \% when approaching $10^{5} \mathrm{~Hz} / \mathrm{cm}^{2}$. These results pave the way towards cost-effective, robust, efficient, large-scale detectors for a variety of applications in future particle, astro-particle and applied fields. A potential target application is digital hadron calorimetry.

(c) 2016 The Authors. Published by Elsevier B.V. This is an open access article under the CC BY-NC-ND
\end{abstract} license (http://creativecommons.org/licenses/by-nc-nd/4.0/).

\section{Introduction}

The Thick Gas Electron Multiplier (THGEM) is a robust radiation detection element suitable for applications requiring large detection areas [1,2]. The broad interest in THGEM-based detectors has resulted in the development of production techniques and concepts (e.g. $[3,4]$ ), including the use of resistive films and materials for reducing occasional discharge effects [5-7]. In this context, the experience gained with various configurations of THGEM-based multipliers with resistive anodes [7-9] has led to the development of a particularly promising candidate - the Resistive-Plate WELL (RPWELL) [10]. It is a single-sided copper-clad THGEM electrode, coupled to a segmented readout anode (pads or strips) through a thin high bulk-resistivity $\left(\sim 10^{8}-10^{10} \Omega \mathrm{cm}\right)$ plate. Extensive laboratory studies in $\mathrm{Ne} /\left(5 \% \mathrm{CH}_{4}\right)$ demonstrated discharge-free operation at high gas-avalanche gains and over a broad ionization range [10]. Good performances in terms of efficiency and average pad multiplicity - studied in the context of future (Semi) Digital Hadronic Calorimeter ((S)DHCAL) [11], were reached, in

\footnotetext{
* Corresponding author.

E-mail address: luca.moleri@weizmann.ac.il (L. Moleri).
}

$\mathrm{Ne} /\left(5 \% \mathrm{CH}_{4}\right)$ with $150 \mathrm{GeV} / \mathrm{c}$ muon and pion beams using a thin $10 \times 10 \mathrm{~cm}^{2}$ RPWELL, with a resistive anode (Semitron ${ }^{\circledR}$ ESD225) of bulk resistivity of $\sim 10^{9} \Omega \mathrm{cm}$ [12]. In the present article we report on new results of further beam studies of this detector, extended to the low-cost $\mathrm{Ar}$-based gas mixtures $\mathrm{Ar} /\left(5 \% \mathrm{CH}_{4}\right)$ and $\mathrm{Ar} /\left(7 \% \mathrm{CO}_{2}\right)$. Detection efficiency values of $\sim 98 \%$ were reached in all conditions, at average pad multiplicity of $\sim 1.2$, in discharge-free operation, also with a high-intensity pion flux. The detector fulfill the requirements for sampling elements in (S)DHCAL and has the potential for applications requiring robust, cheap, efficient largescale detectors with moderate spacial and energy resolutions.

\section{Experimental setup and methodology}

\subsection{RPWELL detector, tracking and readout system}

The $10 \times 10 \mathrm{~cm}^{2}$ RPWELL detector, its SRS/APV25 readout system $[13,14]$ and the experimental setup at the CERN-SPS/H4 beam-line, were detailed in [12] and are briefly described here. The detector scheme, elements and operation principle are shown in Fig. 1. The single-sided THGEM electrode, $0.86 \mathrm{~mm}$ thick, had 
(a)

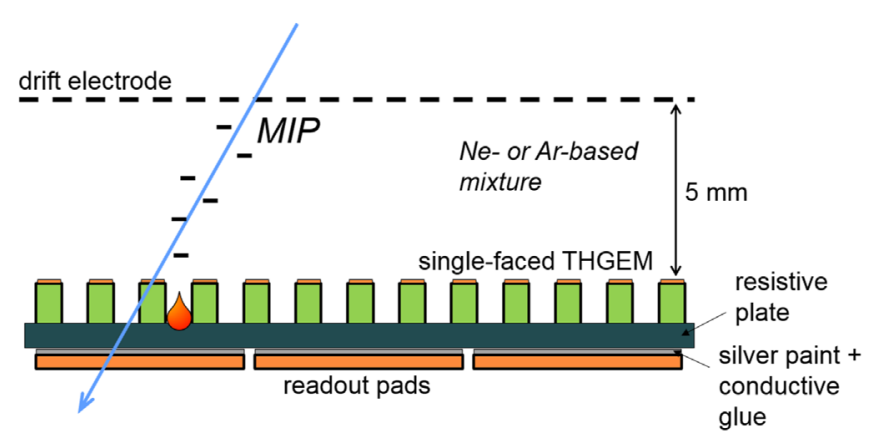

(b)

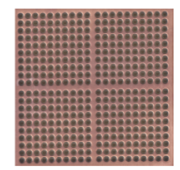

(c)

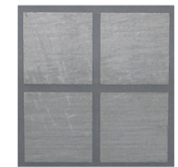

(d)

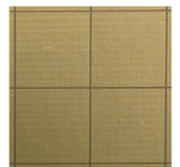

Fig. 1. The $10 \times 10 \mathrm{~cm}^{2}$ RPWELL detector scheme. The RPWELL structure (a): a single sided THGEM (b) coupled to the readout anode through a resistive plate. The anode readout pads (d) are coupled to conductive pads patterned on the resistive plate (c). The metal bands in (b) are located above the underlying pad borders (c,d).

$0.5 \mathrm{~mm}$ diameter holes mechanically drilled in an FR4 plate, copper-clad on one side. The holes were arranged in a square lattice (Fig. 1-b), with $0.96 \mathrm{~mm}$ pitch, so that they cover the underlying $1 \times 1 \mathrm{~cm}^{2}$ anode pads, but not their borders, where $0.86 \mathrm{~mm}$ wide metal bands are left, as described in [8]. The plate was chemically etched, yielding $0.1 \mathrm{~mm}$ rims around the holes, preventing sharp edges and eventual defects. The THGEM electrode was coupled to the anode pads (Fig. 1-d) through a $0.4 \mathrm{~mm}$ thick Semitron ${ }^{\circledR}$ ESD225 ${ }^{1}$ static dissipative plastic plate $\left(2 \times 10^{9} \Omega \mathrm{cm}\right.$ bulk resistivity). The electrical contact between the resistive plate and the readout pads is essential for efficient clearance of the avalanche electrons. Therefore, the bottom of the resistive material was patterned with conductive pads (Fig. 1-c), individually connected to the anode pads (like in [12] but using $3 \mathrm{M}^{\mathrm{TM}}$ Electrically Conductive Adhesive Transfer Tape $972073^{2}$ ).

The detector electrodes were individually biased using CAEN A1833P and A1821N HV power-supply boards, remotely controlled with a CAEN SY2527 unit. The voltage and current in each channel were monitored and stored. All HV inputs were connected through low-pass filters. The RPWELL bias $\left(\Delta V_{\text {RPWELL }}\right)$ with respect to the grounded anode was varied throughout the experiment, while the drift voltage was kept constant: $\Delta V_{\text {drift }}=250 \mathrm{~V}$ - corresponding to a drift field of $\sim 0.5 \mathrm{kV} / \mathrm{cm}$ across the $5 \mathrm{~mm}$ drift gap. The detector was operated in $\mathrm{Ar} /\left(5 \% \mathrm{CH}_{4}\right)$ and $\mathrm{Ar} /\left(7 \% \mathrm{CO}_{2}\right)$ gas mixtures at atmospheric pressure and room temperature, in gas-flow mode (50$100 \mathrm{cc} / \mathrm{min})$; measurements in $\mathrm{Ne} /\left(5 \% \mathrm{CH}_{4}\right)$ were taken for comparison. The operation in the Ar mixtures required higher voltages compared to the ones in $\mathrm{Ne} /\left(5 \% \mathrm{CH}_{4}\right)$ to obtain similar gains. However, with respect to $\mathrm{Ne}$, $\mathrm{Ar}$ has the advantage of larger number of electron-ion pairs produced by a minimum ionizing particle (at normal conditions 94 compared to 39 e-i pairs/cm [15]) - allowing for a smaller drift gap for equal number of primary charges - and considerably lower cost (which might be crucial for large-scale systems). The use of $\mathrm{CO}_{2}$ instead of $\mathrm{CH}_{4}$ as a photon quencher is preferable because the former is non flammable. The triggering, tracking and DAQ system was the same as described in [12]. The RPWELL chambers were placed along the beam line in-

\footnotetext{
${ }^{1}$ www.quadrantplastics.com.

2 www.3m.com.
}

between the tracker elements.

\subsection{Working point: $\triangle \mathrm{V}_{\mathrm{RPWELL}}$, threshold and matching parameter}

As described in [12], for each event the detector pads with signal above threshold were grouped into clusters of neighbors. The global detection efficiency was then calculated as the fraction of particle tracks matched to a cluster in the detector, while the average pad multiplicity was the average number of pads contained in each cluster. The detector working point was adjusted to optimize its performance in each gas mixture, targeting high global detection efficiency at low average pad multiplicity. The lowest-possible value (closest to 1 ) of the latter is a prerequisite for valid particle counting, e.g. in a potential application as a sampling element in DHCAL $[8,16]$. The optimization was done using a set of measurements with $\sim 100 \mathrm{~Hz} / \mathrm{cm}^{2}$ wide $\left(5 \times 5 \mathrm{~cm}^{2}\right)$ muon beam and a $\sim 13,000 \mathrm{~Hz} / \mathrm{cm}^{2}$ narrow $\left(2 \times 2 \mathrm{~cm}^{2}\right)$ pion beam. In both cases, only tracks hitting the detector in a $4 \times 4 \mathrm{~cm}^{2}$ central region were considered. Two important parameters in the analysis are the threshold for zero order suppression (ZSF) and the trackcluster matching parameter $(W)$ (see details in [12]). The values of $\mathrm{ZSF}$ and $\mathrm{W}$ were fixed at $\mathrm{ZSF}=15$ and $\mathrm{W}=10$ or $15 \mathrm{~mm}$ following the same method described in [12].

\section{Results}

\subsection{Detected charge, global detection efficiency and average pad multiplicity}

We studied the detector operation in $\mathrm{Ne} /\left(5 \% \mathrm{CH}_{4}\right), \mathrm{Ar} /\left(5 \% \mathrm{CH}_{4}\right)$ and $\mathrm{Ar} /\left(7 \% \mathrm{CO}_{2}\right)$ using $\sim 100 \mathrm{~Hz} / \mathrm{cm}^{2}$ muon beam. Fig. 2 depicts the most probable value (MPV) of the detected charge, derived from the recorded Landau distributions as a function of $\Delta V_{R P W E L L}$. It is important to note that the effective measured charge is a few times smaller than the avalanche charge; this results from the convolution of the long signal rise time in the RPWELL ( 1-2 $\mu \mathrm{s}$ rise-time) and the short shaping time of the APV25 chip ( 75 ns) as described in [12]. Fig. 3 shows the global detector efficiency as a function of the average pad multiplicity in the same data set shown in Fig. 2. $\triangle \mathrm{V}_{\text {RPWELL }}$ values of $880 \mathrm{~V}, 1640 \mathrm{~V}$ and $1750 \mathrm{~V}$ in

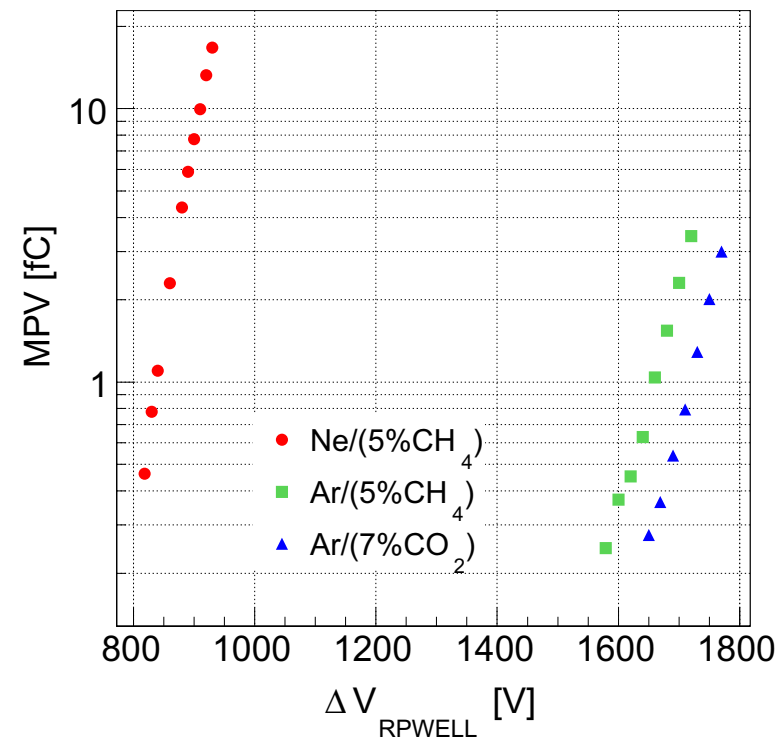

Fig. 2. The most probable value (MPV) of the charge measured by the RPWELL detector in $\sim 100 \mathrm{~Hz} / \mathrm{cm}^{2}$ muon beam for different $\triangle \mathrm{V}_{R P W E L L}$ values in the three gas mixtures. 


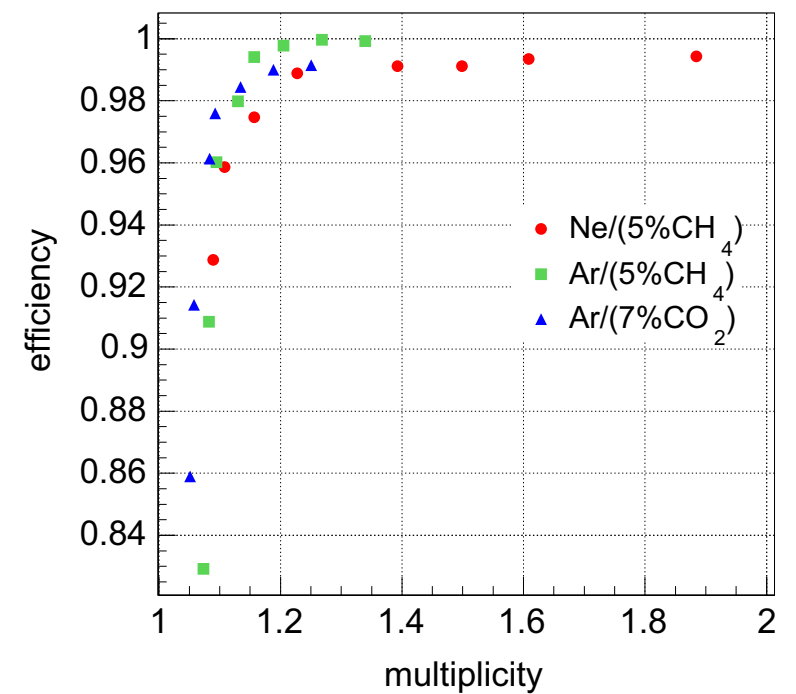

Fig. 3. The global detector efficiency versus average pad multiplicity of the RPWELL detector in $\sim 100 \mathrm{~Hz} / \mathrm{cm}^{2}$ muon beam in the three gas mixtures (using the same data set shown in Fig. 2).

$\mathrm{Ne} /\left(5 \% \mathrm{CH}_{4}\right), \mathrm{Ar} /\left(5 \% \mathrm{CH}_{4}\right)$, and $\mathrm{Ar} /\left(7 \% \mathrm{CO}_{2}\right)$ respectively, gave very high detection efficiency (98-99\%) and low pad multiplicity $(\sim 1.2)$ in a stable condition.

\subsection{Performance under low and high particle flux}

The RPWELL performance was investigated with low-rate muon and high rate pion beams reaching a flux of $\sim 4 \cdot 10^{5} \mathrm{~Hz} / \mathrm{cm}^{2}$. The results are shown in Fig. 4. Note that in order to keep a high efficiency at high particle fluxes, these measurements were done using higher voltages than those optimal for detecting low-rate muons. The values of $\Delta \mathrm{V}_{\text {RPWELL }}$ were $880 \mathrm{~V}, 1700 \mathrm{~V}$ and $1770 \mathrm{~V}$ in $\mathrm{Ne} /\left(5 \% \mathrm{CH}_{4}\right), \mathrm{Ar} /\left(5 \% \mathrm{CH}_{4}\right)$ and $\mathrm{Ar} /\left(7 \% \mathrm{CO}_{2}\right)$ respectively. The global detection efficiency (Fig. 4) is stable until rates of $\sim 10^{4} \mathrm{~Hz} / \mathrm{cm}^{2}$, consistently for all three gas mixtures. It drops by a few \% (to $94 \%$ ) while approaching rates of $\sim 10^{5} \mathrm{~Hz} / \mathrm{cm}^{2}$, due to $30 \%$ gain loss (not shown), possibly resulting from the charging up of the holes and avalanche build-up limitations on the resistive anode (see for example [17]). These results are in agreement with that previously

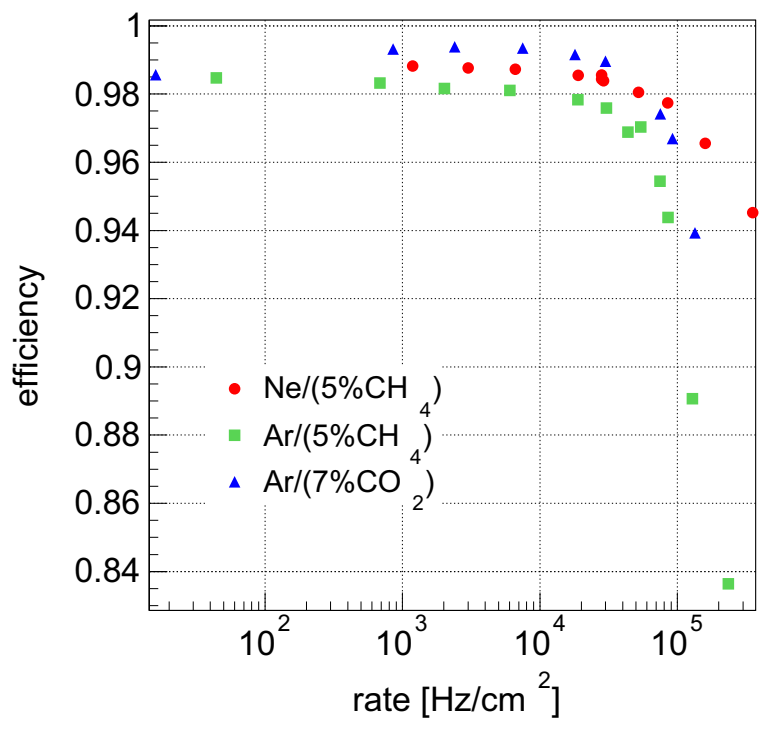

Fig. 4. Global detection efficiency of the RPWELL detector as a function of the incoming particle flux in $\mathrm{Ne} /\left(5 \% \mathrm{CH}_{4}\right), \mathrm{Ar} /\left(5 \% \mathrm{CH}_{4}\right)$ and $\mathrm{Ar} /\left(7 \% \mathrm{CO}_{2}\right)$. The values of $\triangle \mathrm{V}_{R P W E L L}$ were $880 \mathrm{~V}, 1700 \mathrm{~V}$ and $1770 \mathrm{~V}$ respectively.

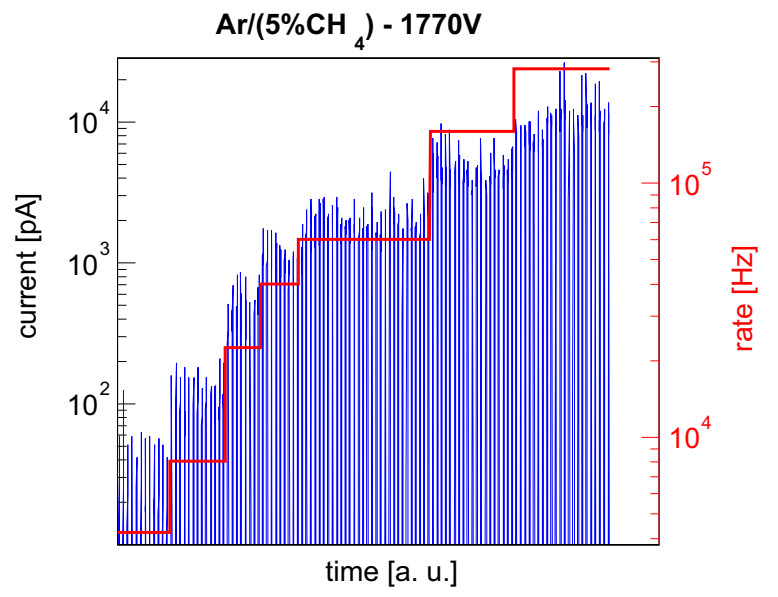

Fig. 5. Current flowing through the detector during pion runs at different rates in $\mathrm{Ar} /\left(5 \% \mathrm{CH}_{4}\right)$. The beam spill-structure is clearly visible.

shown in $\mathrm{Ne} /\left(5 \% \mathrm{CH}_{4}\right)[12]$. The efficiency drop can be mitigated using higher operation voltage.

To demonstrate the electrical stability of the RPWELL, we measured the current flowing through the anode using a sensitive ammeter [18], while irradiating the detector with pions at different rates. Fig. 5 shows the current and the pion rates as a function of time. The measurement shown is in $\operatorname{Ar} /\left(7 \% \mathrm{CO}_{2}\right)$, and similar results were obtained in all three gas mixtures. As expected, the small current spikes, corresponding to the beam spill-structure, grow smoothly in amplitude with the particle rate. The measured value of the current should follow the simple expression: $\mathrm{I}=q \cdot n \cdot \Phi \cdot G(\Phi)$, where $I$ is the current, $q$ is the electron charge, $n$ is the number of electron-ion pairs produced by a minimum ionizing particle in $5 \mathrm{~mm}$ of $\operatorname{Ar}$ [15], $\Phi$ is the particle rate and $G$ the detector gain (which depends on $\Phi$ as explained above). Reversing the formula and using the measured value of the current, we can estimate the value of $G$. For example for $\Phi=4 \cdot 10^{3} \mathrm{~Hz}$ we get $G=1.3 \times 10^{3}$, which is compatible with the value obtained from the measured charge MPV (Fig. 2 which is taken at a similar particle rate), once taking into account the effect of the electronics shaping time.

\subsection{Gain stability over time}

A stable operation of the detector was demonstrated over time, under $10^{4}-10^{5} \mathrm{~Hz} / \mathrm{cm}^{2}$ pion fluxes, as shown in Fig. 6 . The applied voltages were the same as those of the measurements presented in Section 3.2. No significant gain variations (less than $5 \%$ ) were observed along $\sim 1 \mathrm{~h}$ of operation in all three gas mixtures. The values of global detection efficiency and average pad multiplicity during these measurements also remained stable.

\subsection{Discharge probability}

A discharge was defined as an abrupt increase in the current supplied to the detector [12]. Discharge probability was measured during the high-rate pion runs presented in Section 3.3. No discharges were observed in any of the gas mixtures while irradiating the detector with over $10^{8}$ pions; therefore the resulting value of $10^{-8}$ is an upper limit for the discharge probability in the present RPWELL configuration. Since pions are prone to induce highlyionizing secondary events, we have an additional indication on the broad dynamic range of this detector. 


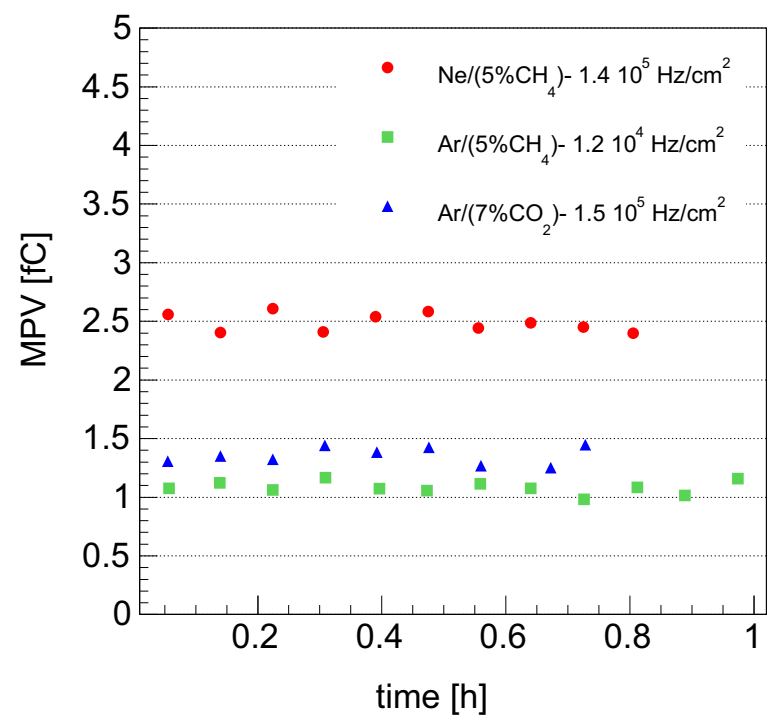

Fig. 6. Gain stability over time under a high-rate $\left(10^{4}-10^{5} \mathrm{~Hz} / \mathrm{cm}^{2}\right)$ pion flux in $\mathrm{Ne} /\left(5 \% \mathrm{CH}_{4}\right), \mathrm{Ar} /\left(5 \% \mathrm{CH}_{4}\right)$ and $\mathrm{Ar} /\left(7 \% \mathrm{CO}_{2}\right)$. The values of $\Delta \mathrm{V}_{R P W E L L}$ were $880 \mathrm{~V}, 1700 \mathrm{~V}$ and $1770 \mathrm{~V}$ respectively.

\section{Summary and discussion}

A $6 \mathrm{~mm}$ thick (without readout) $10 \times 10$ RPWELL detector with a Semitron ${ }^{\circledR}$ ESD225 resistive plate coupled to a pad readout anode was investigated for the first time in $\operatorname{Ar} /\left(5 \% \mathrm{CH}_{4}\right)$ and $\operatorname{Ar} /\left(7 \% \mathrm{CO}_{2}\right)$ gas mixtures; its performance was compared with that in $\mathrm{Ne} /\left(5 \% \mathrm{CH}_{4}\right)$. This thin, single-stage detector was operated with $150 \mathrm{GeV} / \mathrm{c}$ muons and pions, at fluxes reaching $4 \cdot 10^{5} \mathrm{~Hz} / \mathrm{cm}^{2}$. High detection efficiency values, greater than $98 \%$, at low average pad multiplicity of $\sim 1.2$, were demonstrated in all three gas mixtures, maintaining stable, discharge-free operation, also at high pion flux. The efficiency remained unaffected up to a pion flux of $10^{4} \mathrm{~Hz} / \mathrm{cm}^{2}$, above which it decreased by a few $\%$ at $\sim 10^{5} \mathrm{~Hz} / \mathrm{cm}^{2}$. For example, in $\operatorname{Ar} /\left(7 \% \mathrm{CO}_{2}\right)$ the RPWELL maintained a global detection efficiency of $95 \%$ under a flux of $1.5 \cdot 10^{5} \mathrm{~Hz} / \mathrm{cm}^{2}$ pions. The lack of electrical instabilities over more than $10^{8}$ pion events sets an upper limit of $10^{-8}$ on the discharge probability $-\sim 2$ orders of magnitude better than other THGEM-based configurations (e.g. single- and double-THGEM, Resistive WELL and Segmented Resistive WELL reviewed in [7]).

Compared to other detector technologies explored under similar conditions, e.g. for the DHCAL, the performance of the $10 \times 10 \mathrm{~cm}^{2}$ RPWELL detector, with respect to detection efficiency and pad multiplicity, is superior to that of $1 \times 1 \mathrm{~m}^{2}$ RPCs $[17,19]$ and $30 \times 30 \mathrm{~cm}^{2}$ GEM detectors [20]; it is similar to that of $1 \times 1 \mathrm{~m}^{2}$ MICROMEGAS [21,22]. The efficiency dependence on the incoming particle flux is similar to that of multi-gap RPCs based on semi-conductive glass [23]. Also in terms of electrical stability the performance of the RPWELL detector is similar to that of RPCs. These results pave the way towards robust, efficient large-scale detectors for applications requiring economic solutions at moderate spatial and energy resolutions. A different application than digital counting (like in DHCAL or RICH) could be that of a tracking detector with strip readout. In this case it would require charge spreading over a resistive layer to increase the spatial resolution (see for example [24]). This detector configuration, as well as the RPWELL technology scale-up and the investigation of other suitable resistive materials are the subject of current R\&D.

\section{Acknowledgments}

This project has received funding from: the European Unions Horizon 2020 Research and Innovation Program under grant agreement No. 654168; I-CORE Program of the Planning and Budgeting Committee and The Israel Science Foundation (grant No. 1937/12); Nella and Leon Benoziyo Center 220 for High Energy Physics. A. Breskin is the W.P. Reuther Professor of Research in the Peaceful use of Atomic Energy. F.D. Amaro and C.D.R. Azevedo acknowledge support by FCT under Post-Doctoral Grant SFRH/ BPD/74775/2010 and SFRH/BPD//79163/2011. Work done within the CERN RD51 framework.

\section{References}

[1] R. Chechik, et al., Thick gem-like hole multipliers: properties and possible applications, Nucl. Instrum. Methods A 535 (2004) 303-308.

[2] A. Breskin, et al., A concise review on thgem detectors, Nucl. Instrum. Methods A 598 (2009) 107-111.

[3] Q. Liu, et al., A successful application of thinner-thgems, JINST 8 (2013) C11008.

[4] M. Alexeev, et al., Ion backflow in thick gem-based detectors of single photons, JINST 8 (2013) P01021.

[5] V. Peskov, et al., Development and first tests of gem-like detectors with resistive electrodes, IEEE Trans. Nucl. Sci. 54 (2007) 1784-1791.

[6] G. Charpak, et al., Progress in the development of a s-retgem-based detector for an early forest fire warning system, JINST 4 (2009) P12007.

[7] S. Bressler, et al., Recent advances with thgem detectors, JINST 8 (2013) C12012.

[8] L. Arazi, et al., Thgem-based detectors for sampling elements in dhcal: laboratory and beam evaluation, JINST 7 (2012) C05011.

[9] L. Arazi, et al., Laboratory studies of thgem-based well structures with resistive anode, JINST 9 (2014) P04011.

[10] A. Rubin, et al., First studies with the Resistive-Plate WELL gaseous multiplier, JINST 8 (2013) P11004.

[11] T. Behnke, et al. The international linear collider technical design report-volume 4: Detectors, arXiv preprint arXiv:1306.6329.

[12] S. Bressler, et al., First in-beam studies of a Resistive-Plate WELL gaseous multiplier, JINST 11 (2016) P01005.

[13] S. Martoiu, et al., Development of the scalable readout system for micro-pattern gas detectors and other applications, JINST 8 (2013) C03015.

[14] M.J. French, et al., Design and results from the apv25, a deep sub-micron cmos front-end chip for the cms tracker, Nucl. Instrum. Methods A 466 (2001) 359-365.

[15] F. Sauli, Gaseous Radiation Detectors: Fundamentals and Applications, Cambridge University Press, 2014.

[16] F. Sefkow, et al. Experimental tests of particle flow calorimetry, arXiv preprint arXiv:1507.05893.

[17] M. Affatigato, et al. Measurements of the rate capability of various resistive plate chambers, JINST 10 (2015) P10037.

[18] H. Müller, CERN, presented in rd51 miniweek, 2015.

[19] B. Bilki, et al., Calibration of a digital hadron calorimeter with muons, JINST 3 (2008) P05001.

[20] J. Yu, et al., Application of large scale gem for digital hadron calorimetry, Phys. Procedia 37 (2012) 393-401.

[21] C. Adloff, et al., Micromegas chambers for hadronic calorimetry at a future linear collider, JINST 4 (2009) P11023.

[22] M. Chefdeville, et al., Micromegas for sampling calorimetry, PoS (2014) 054

[23] L. Naumann, et al., Ceramics high rate timing RPC, Nucl. Intrum. Methods A 628 (2011) 138-141.

[24] M. Cortesi, et al., Investigations of a THGEM-based imaging detector, JINST 2 (2007) P09002. 